\title{
Changes in the axon terminals of primary afferents from a single vibrissa in the rat trigeminal nuclei after active touch deprivation or exposure to an enriched environment
}

\author{
Julia Fernández-Montoya ${ }^{+1}$, Yasmina B. Martin ${ }^{+2}$, Pilar Negredo ${ }^{1}$ and Carlos Avendaño*1 \\ ${ }^{1}$ Department of Anatomy, Histology and Neuroscience, Autonoma University of Madrid, \\ Medical School, Madrid, Spain \\ ${ }^{2}$ Department of Anatomy, Health Sciences School, Francisco de Vitoria University, Pozuelo de \\ Alarcón, Spain \\ + The first two authors contributed equally to this work.
}

Julia Fernández-Montoya, B.Sc., M.Sc.

Departamento de Anatomía, Histología y Neurociencia

Facultad de Medicina

Universidad Autónoma de Madrid

c/ Arzobispo Morcillo 2

28029 Madrid (Spain)

Ph: +34 914972114

Email: julia.fernandez@inv.uam.es

Yasmina B. Martin, Ph.D.

Departamento de Anatomía

Facultad de Ciencias de la Salud

Universidad Francisco de Vitoria

28223 Pozuelo de Alarcón, Madrid (Spain)

Ph: +34 913510303

Email: y.martin.prof@ufv.es

Pilar Negredo, Ph.D.

Departamento de Anatomía, Histología y Neurociencia

Facultad de Medicina

Universidad Autónoma de Madrid

c/ Arzobispo Morcillo 2

28029 Madrid (Spain)

Ph: +34 914975354

Email: pilar.negredo@uam.es

*Corresponding author:

Carlos Avendaño, M.D., Ph.D.

Departamento de Anatomía, Histología y Neurociencia

Facultad de Medicina

Universidad Autónoma de Madrid

c/ Arzobispo Morcillo 2

28029 Madrid (Spain)

Ph: +34 914975335

Email: carlos.avendano@uam.es 
Keywords Isolectin B4 · Primary afferent · Input-dependent plasticity · Trigeminal ganglion · Transganglionic

$\begin{array}{ll}\text { Abbreviations } \\ \text { ABC } & \text { Avidin-Biotynilated peroxidase Complex } \\ \text { Cb } & \text { Cerebellum } \\ \text { CE } & \text { Coefficient of error } \\ \text { CTB } & \text { Cholera toxin, fraction B } \\ \text { DAB } & \text { 3,3'-Diaminobenzidine } \\ \text { DVN } & \text { Deep vibrissal nerve } \\ \text { DRG } & \text { Dorsal root ganglion } \\ \text { HRP } & \text { Horseradish peroxidase } \\ \text { IB4 } & \text { Isolectin B4 from Griffoniasimplicifolia } \\ \text { IoN } & \text { Infraorbital nerve } \\ \text { PB } & \text { Phosphate buffer } \\ \text { Pr5 } & \text { Principal trigeminal nucleus } \\ \text { Sp5c } & \text { Spinal trigeminal nucleus, pars caudalis } \\ \text { Sp5ip } & \text { Spinal trigeminal nucleus, pars interpolaris } \\ \text { Sp5o } & \text { Spinal trigeminal nucleus, pars oralis } \\ \text { t5 } & \text { Spinal trigeminal tract } \\ \text { TG } & \text { Trigeminal ganglion } \\ \text { WGA } & \text { Wheat germ agglutinin }\end{array}$




\begin{abstract}
Lasting modifications of sensory input induce structural and functional changes in the brain, but the involvement of primary sensory neurons in this plasticity has been practically ignored. Here we examine qualitatively and quantitatively the central axonal terminations of a population of trigeminal ganglion neurons whose peripheral axons innervate a single mystacial vibrissa. Vibrissa follicles are heavily innervated by myelinated and unmyelinated fibers that exit the follicle mainly through a single deep vibrissal nerve (DVN). We made intraneural injections of a mixture of cholera-toxin B (CTB) and isolectin B4, tracers for myelinated and unmyelinated fibers respectively, in three groups of young adult rats: controls, animals subjected to chronic haptic touch deprivation by unilateral whisker trimming, and rats exposed for two months to environmental enrichment. The regional and laminar pattern of terminal arborizations in the trigeminal nuclei of the brain stem did not show gross changes after sensory input modification. However, there were significant and widespread increases in the number and size of CTB-labeled varicosities in the enriched condition, and a prominent expansion in both parameters in laminae III-IV of the caudal division of the spinal nucleus in the whisker trimming condition. No obvious changes were detected in IB4-labeled terminals in laminae I-II. These results show that a prolonged exposure to changes in sensory input without any neural damage is capable of inducing structural changes in terminals of primary afferents in mature animals, and highlight the importance of peripheral structures as the presumed earliest players in sensory experience-dependent plasticity.
\end{abstract}

\title{
Introduction
}

Rats use their facial macrovibrissae (mystacial vibrissae or whiskers) to gather multiple aspects of tactile information by actively sensing their surroundings with rhythmic sweeping movements (whisking) that allow the animal to navigate and explore physical properties and location of objects. The features of this system have proved ideal for investigating somesthesia and sensorimotor control and integration in mammals (reviews in Diamond 1988; Kleinfeld 2009; Bush et al. 2016). Each whisker follicle is heavily innervated by myelinated and unmyelinated fibers ending in a variety of sensory terminals and receptors, which leave the follicle by either of two largely segregated routes: two or more small superficial vibrissal nerves, or a larger and single deep vibrissal nerve (DVN; Rice et al. 1986). Together with other afferents from intervibrissal skin, hair follicles, teeth and oral mucosa these nerves are the peripheral divisions of pseudomonopolar primary sensory neurons in the trigeminal ganglion (TG) that reach the face via the infraorbital nerve (IoN).

The central projections from TG neurons distribute in well-defined spatial patterns in the trigeminal nuclei of the brain stem. The dorsoventral and mediolateral location, as well as the morphology of the terminals of primary afferents from the whiskers largely depend on the type and location of their peripheral receptive fields. Afferents from the vibrissae follicles were found to terminate forming a continuous rostrocaudal column through the principal trigeminal nucleus (Pr5), and the oral (Sp5o) and interpolar (Sp5ip) divisions of the spinal trigeminal nucleus (Arvidsson 1982; Arvidsson and Rice 1991). Terminations in the caudal division (Sp5c) in addition displayed a laminar pattern (Hayashi 1980; Jacquin et al. 1986), resembling that of cutaneous afferents in the spinal cord, where large myelinated, A $\beta$ low-threshold mechanoreceptors distribute in laminae III-IV, thinly myelinated A $\delta$ afferents end in laminae I and $\mathrm{V}$, and unmyelinated $\mathrm{C}$-fiber thermo- and nociceptors remain restricted to laminae I and II (for review see Brown 1981 and Willis and Coggeshall 2004). 
The pattern of primary afferent terminals from spinal nerves can be altered following injury to the peripheral nerves. Even in mature animals, when the damage is severe and is soon followed by degenerative phenomena, regenerative changes, such as collateral sprouting from surviving axons, take place at later stages (Aldskogius et al. 1985; Woolf et al. 1992; Woolf et al. 1995; Florence and Kaas 1995; Bao et al. 2002). Similar studies in the trigeminal system are scarce, and early reports on translaminar expansion of afferents in Sp5c after IoN transection have proven not to be conclusive (Bjelke et al. 1996; Arvidsson and Johansson 1988; Zhang et al. 2015). Unsurprisingly, studies are lacking on the anatomy of primary afferent terminals under conditions of sustained alterations of the sensory input in absence of damage to peripheral tissues, receptors or nerves. However, we found recently that sensory deprivation by repeated whisker trimming, or long-term exposure to an enriched environment in adult rats induce modifications in glutamatergic transmission-related gene expression and protein levels in the TG (Fernandez-Montoya et al. 2016). In previous studies we had also shown that unilateral deprivation by chronic whisker trimming provoked bilateral changes in the length, number and distribution of dendrites and dendritic spines of trigeminothalamic barrelette neurons of Pr5, and intersubnuclear neurons of Sp5c (Negredo et al. 2009; Martin et al. 2014).

In the present study we investigated whether the axon terminals of the TG primary sensory neurons developed structural adjustments following chronic whisker trimming or exposure to an enriched environment. We injected in the DVN of vibrissa $\mathrm{C} 1$ a mixture of the fraction $\mathrm{B}$ of cholera toxin (CTB), a tracer that is taken up by myelinated fibers (LaMotte et al. 1991; Robertson et al. 1991), and the plant isolectin B4 (IB4), considered a selective marker of unmyelinated fibers (Ambalavanar and Morris 1993; Silverman and Kruger 1990), to study the distribution of primary afferents in the brain stem trigeminal nuclei. Our results showed that a prolonged exposure to a novel or altered pattern of sensory input, without any nerve or receptor injury, is capable of inducing patent morphometric changes in those terminals. These findings add support to the notion that experience-dependent anatomical plasticity in mature mammals already occurs at the earliest processing steps in the sensory pathways.

\section{Materials and Methods}

\section{Experimental animals}

Young adult male Sprague-Dawley rats $(n=26)$ from our own colony, originating from Harlan (Harlan Iberica, Barcelona, Spain) were used. All animal procedures were approved in advance by the Ethical Committee of the Universidad Autonoma de Madrid, in accordance with the European Community's Council Directive 2010/63/UE. Every effort was made to minimize the suffering of the animals, as well as the number of animals used.

The animals were divided into three groups: 1) Control animals (Group C, $n=14$ ) included rats that were left untouched, except for routine cleaning and feeding procedures, until the end of the experiment. 2) Deprived animals (Group T, $n=6$ ), rats that were subjected to a chronic (7-8 weeks) deprivation of active (haptic) touch by trimming all whiskers in the right side every 2-3 days from the beginning of the third month of life. Trimming was performed on hand-held animals so that regrown vibrissae never exceeded $3 \mathrm{~mm}$ (Ebner 2005); care was taken to avoid hair plucking or skin damage during the procedure. 3) Animals exposed to an enriched environment (Group E, n=6) 4 h/day, 5 days/week for 7-8 weeks. For achieving this "sensoryenriched" condition two groups of eight animals each were placed in a large cage $(100 \mathrm{~cm} \times 80$ $\mathrm{cm} \times 60 \mathrm{~cm}$ ) with a variety of natural and artificial objects of different textures, which were changed every 5 th day (four animals of each group were used for a separate study). Not more than two littermates from the same dam were used in each group. 
Except for the periods of specific manipulations, all rats were housed under standard colony conditions (4 rats per cage). Food and water were supplied ad libitum, and the animals were kept under a reversed 12:12 h dark/light cycle.

\section{Surgery and fixation}

All surgeries were performed under a mixture of ketamine $55 \mathrm{mg} / \mathrm{Kg}$, xylazine $15 \mathrm{mg} / \mathrm{Kg}$ and atropine $0.2 \mathrm{mg} / \mathrm{Kg}$ in aseptic conditions, and under a surgical microscope (Zeiss-Jena). After identifying the $\mathrm{C} 1$ whisker, a small incision was made just behind the vibrissal pad. The skin edges were separated and the target whisker follicle and the outgoing DVN were exposed. Both were gently separated from the surrounding connective tissue, and placed on a narrow pliable metal plate ensuring that the nerve was not stretched. A solution containing a mixture of $2 \%$ CTB (Sigma Aldrich), 2\% IB4 (Vector Laboratories) and traces of the supravital stain Light Green was freshly prepared. With the aid of a micromanipulator, a glass micropipette (A-M Systems, Sequim, WA; 20-30 $\mu \mathrm{m}$ tip outer diameter) coupled to a Hamilton syringe was filled with the solution and used to slowly inject 1-1.5 $\mu$ l into the C1 DVN in 3-4 pulses spaced over a 30-40 min period (Suppl. Fig. 1A).

Four days $(\mathrm{n}=22)$ or $24 \mathrm{~h}(\mathrm{n}=3)$ after DVN injection, rats were deeply anesthetized (Dolethal, $50 \mathrm{mg} / \mathrm{kg}$ i.p.) and perfused through the ascending aorta with $0.9 \% \mathrm{NaCl}(100 \mathrm{ml}, 2$ minutes), followed by $4 \%$ paraformaldehyde in $0.1 \mathrm{M}$ phosphate buffer (PB; $\mathrm{pH} 7.4$ ). The brain stems of rats with longer postinjection survival time, and the TG and DVN of rats with $24 \mathrm{~h}$ survival were removed and postfixed in the same fixative overnight at $4^{\circ} \mathrm{C}$. Except for the DVN in two cases that would be used for preembedding immunohistochemistry (see below), all the tissue was then cryoprotected for 2 days in $30 \%$ sucrose in $0.1 \mathrm{M}$ PB. In addition, the DVN from a naïve, uninjected rat, was postfixed for two days with a buffered mixture of $2 \%$ paraformaldehyde and $2.5 \%$ glutaraldehyde.

\section{Immunohistochemistry}

The brain stems and TGs were frozen and serially cut at $40 \mu \mathrm{m}$ in the horizontal plane in a sliding microtome. Brain stem sections were processed free-floating using the avidin-biotin peroxidase method (ABC), with diaminobenzidine (DAB) as a chromogen. Endogenous peroxidase was inactivated by incubating sections in a solution of $1 \%$ hydrogen peroxide in $0.1 \mathrm{M}$ PB for 10 minutes. Sections were then incubated in $5 \%$ normal goat or rabbit serum in PBS with 2\% Triton ${ }^{\mathrm{TM}}$ X-100 (Sigma Aldrich) for 2 hours. Alternate series were incubated either with goat anti-IB4 $(1 \mu \mathrm{g} / \mathrm{ml}$; Vector Laboratories), or with rabbit anti-CTB (1:500; Sigma Aldrich), overnight at $4^{\circ} \mathrm{C}$. The next day, sections were incubated, respectively, in rabbit antigoat (1:250, Vector Laboratories) and goat anti-rabbit (1:500, Sigma-Aldrich) biotinylated secondary antibodies at room temperature for $2 \mathrm{~h}$, followed by ABC (1:100; Kit ABC Elite $®$, Vector Laboratories) at room temperature for $2 \mathrm{~h}$ and revealed with DAB $(0.05 \%$ in PB $0.1 \mathrm{M}$; Sigma Aldrich) with $\mathrm{H}_{2} \mathrm{O}_{2}$ added ( $0.003 \%$ of the stock solution $\left.33 \%\right)$. The sections were examined and photographed in an Olympus BX61 microscope.

TG sections were serially mounted on glass slides and immunoreacted in a moist chamber. After endogenous peroxidase inhibition sections were preincubated in 5\% horse normal serum in PBS with 2\% Triton ${ }^{\mathrm{TM}} \mathrm{X}-100$ (Sigma Aldrich) for $2 \mathrm{~h}$. Sections were then incubated with a mixture of the two primary antibodies, anti-IB4 $(1 \mu \mathrm{g} / \mathrm{ml}$; Vector Laboratories) and anti-CTB (1:500; Sigma Aldrich), overnight at $4^{\circ} \mathrm{C}$. After several washes, sections were exposed to a mixture of the secondary biotinylated antibodies, rabbit anti-goat (1:250, Vector Laboratories) and goat anti-rabbit (1:500, Sigma-Aldrich) for $2 \mathrm{~h}$ at room temperature. This was followed by the $\mathrm{ABC}$ and $\mathrm{DAB}$ steps as indicated for the brain stems. 
Thin cross-section slices of the DVN were processed free-floating with the same protocol as that applied to the brain stems, although reducing the concentration of Triton ${ }^{\mathrm{TM}} \mathrm{X}-100$ (Sigma Aldrich) to $0.025 \%$. After the immunoreaction, sections were osmicated, dehydrated and embedded in Durcupan® ACM resin (Fluka AG, Buchs, Switzerland).

\section{Electron microscopy}

Semithin and ultrathin sections of the DVN were obtained using a Leica Ultracut UCT ultramicrotome (Leica Microsystems AG, Wetzlar) and a diamond knife (Drukker International B.V., Cuijk, The Netherlands). The ultrathin sections were collected on 100-mesh nickel grids and stained with uranyl acetate and lead citrate. The concentration and time of exposure to lead citrate was varied, so that stronger staining was achieved in the naïve control case, and a weaker staining was obtained in the immunoreacted cases. Some immunoreacted semithin sections were counterstained with toluidine blue. Digital ultrastructural images were captured at 500X or 8000X in a Jeol 1010 electron microscope (Tokyo, Japan) equipped with a CCD camera (Gatan BioScan, Pleasanton, CA, USA).

\section{Identification of varicosities}

CTB-labeled profiles that were defined as varicosities ranged from small spheroidal dilatations along barely stained thin axons to large ovoid or irregular swellings (Fig. 1). Since varicosities in very thin axons presumably remain beyond resolution in optical microscopy, it is expected that their identification resulted in a systematic underestimation bias when counting. Also, tagging a small labeled profile as varicosity risks bias due to undercounting (from skipping all small profiles and losing those obscured by larger ones) or overcounting (from including in the count cross-cut labeled axonal profiles not necessarily varicose). In order to be able to demarcate a population of varicosities that could be counted in an unbiased, consistent and reproducible manner, varicosities were classified into two populations, $d$ and $q$, according to the following criteria: distinct varicosities included large and medium-sized profiles, which are easily identifiable, plus those labeled profiles that, albeit small, represented swellings at least twice the diameter of a visible parent axon. Separate analyses of the same samples by two researchers (JFM and CA) yielded a remarkable degree of coincidence (values differing by about $5 \%$ over more than two thousand $d$ varicosities analyzed on the same sections, with independent samplings). A second, questionable population of varicosities included isolated, dot-like profiles not obviously associated to a visible axon. In all likelihood, this $q$ population carried the bias due to signal loss or weakness and overlapping (Avendaño 2006), and the ability to distinguish these small varicosities was heavily dependent on experience.

\section{Double immunofluorescence}

Eight micron-thick cross sections of one C1 DVN and one TG, and $40 \mu \mathrm{m}$-thick horizontal sections of the brain stem from two animals were obtained in a cryostat and a sliding microtome, respectively, and were used for immunofluorescence. All tissue was preincubated in $10 \%$ normal donkey serum in PBS with $2 \%$ Triton $^{\mathrm{TM}} \mathrm{X}-100$ (Sigma Aldrich) for $2 \mathrm{~h}$. This was followed by incubation overnight in a combination of two primary antibodies, goat antiIB4 (1 $\mu \mathrm{g} / \mathrm{ml}$; Vector Laboratories) and rabbit anti-CTB (1:500; Sigma Aldrich). After appropriate washes, the tissue was incubated for $2 \mathrm{~h}$ in the dark in a mixture of two speciesspecific secondary antibodies: donkey anti-goat AlexaFluor®488, and donkey anti-rabbit AlexaFluor ${ }^{\circledR} 647$ (1:200; Invitrogen $\left.{ }^{\mathrm{TM}}\right)$. The sections were mounted in Vectashield mounting medium for fluorescence with DAPI (Vector Laboratories). Prior to analysis with the confocal 
microscope, the location and quality of the immunofluorescence for CTB and IB4 was assessed with the epifluorescence equipment integrated in an Olympus BX61 microscope, using appropriate lenses and excitation filters.

\section{Confocal microscopy and colocalization analysis}

Confocal images were obtained using a TCS SP5 Spectral Leica Confocal microscope using an oil-immersion 40X lens for low-power images of Sp5c, and a 2x zoom for resolving colocalization of tracers at the single ax on level. For each field, images were obtained with a sequential acquisition setting at $1024 \times 1024$ pixels. For high power observation, 12-14 optical sections were scanned at $0.5 \mu \mathrm{m}$ z-separation. The colocalization of CTB and IB4 was examined in single $0.5 \mu \mathrm{m}$ optical sections using orthogonal (xy, xz, yz) views. Image stacks and merged channel panels were then prepared with Leica LAS AF software.

\section{Stereology}

The quantitative study of the varicosities was performed blind to the group, and the data analysis was not initiated before all data were collected. Three sets of measurements were collected separately from each trigeminal nucleus to assess the spread and intensity of the innervation by the primary afferents from the DVN: (1) The volume of the neuropil reached by axonal varicosities was estimated by point counting and the Cavalieri principle (Gundersen et al. 1988), both on CTB- and IB4-immunolabeled sections. (2) The absolute number of CTBlabeled varicosities, as well as of labeled neurons in the TG was estimated using the optical fractionator (West et al. 1991). And (3) the distribution of these varicosities according to their apparent cross-sectional size was estimated by means of the 2D nucleator (Gundersen 1988; Larsen 1998). All measurements were performed in an integrated stereological setup consisting of a BX61 Olympus microscope connected to an Olympus DP71 high-resolution video camera (Olympus-Europa, Hamburg, Germany), and a high-precision motorized microscope stage (Prior Proscan II, Prior Scientific Inc., Rockland, MA) that included a $0.1 \mu \mathrm{m}$ resolution z-axis encoder. The interactive test grids and the control of the motorized stage were provided by the NewCAST stereological software package (Visiopharm, Hørsholm, Denmark).

For nuclear volume estimation the tissue was sampled with a quadratic lattice of points, and a planapochromatic $20 \mathrm{X}$ dry objective (Olympus UPLSAPO, NA=0.75) was used to identify CTB-labeled axonal varicosities. Points were counted whenever a varicosity fell within a $4 \mu \mathrm{m}$ range of a point anywhere across the tissue depth. IB4 immunoreactivity resulted in a more diffuse labeling of axonal profiles peppered with small varicosities that were clearly outlined only at zones of heavy staining, so that delineation of the immunolabeled territory was less precise at the more weakly stained periphery. The lattice was laid systematically over the whole labeled territory and the point counts were used for estimating volume according to the classical equation: $\widehat{V}=\Sigma P \cdot a(p) \cdot \bar{d}$ where the sum of points counted is multiplied by the area associated to each sampling point (in our case, $\mathrm{a}(\mathrm{p})=3040 \mu \mathrm{m}^{2}$ ) and the mean distance between two consecutively studied sections (here, $\mathrm{d}=80 \mu \mathrm{m}$ ) to estimate the tissue volume occupied by labeled varicosities. Values were not corrected for shrinkage.

For varicosity counting and measuring, a planapochromatic $100 \mathrm{X}$ oil immersion lens (Olympus UPLSAPO, NA=1.4) was used. A fractionator sampling scheme was applied in three successive stages: section series (all CTB-immunoreacted sections were used), section area, and section depth. The apparent "equator" of the varicosity (plane at which the varicosity displayed maximum sharpness at its periphery) was used as the counting unit. The completeness of immunolabeling throughout the depth of the section was assessed in all regions. When poor staining was detected in the central $1 / 3$ or $1 / 4$ of the section, this stretch of 
the tissue was excluded from counting. To avoid the potential estimation bias due to variability in section thickness, the contribution of each section to the final number estimate was systematically weighted by the number of varicosities counted in each section (Avendaño 2006). Excluding lamina II in Sp5c, an average of 736 varicosities was counted per nucleus and case, from a minimum of 270 (in Sp5o of controls) to a maximum of 1058 (in Sp5c of group $\mathrm{T})$. From these direct counts, the total number of varicosities in each nucleus was then estimated as: $\widehat{N}=\Sigma Q^{-} \cdot F_{T}{ }^{-1}$ where $\Sigma Q^{-}$is the number of varicosities counted, and $F_{T}{ }^{-1}$ is the inverse of the final or total fraction (in our cases, on average, 1:56).

The optical fractionator design was also applied to estimate the total number of labeled neurons in the TG. Since the expected number was small, the sampling intensity was high, so that the total fraction was about 1:12.

The precision of the estimations of $\mathrm{V}$ and $\mathrm{N}$ was assessed by estimating the coefficient of error (CE) as described for systematic random samples (Cruz-Orive 1999). The CEs obtained for the estimations of the volume of nuclear territories displaying varicosities ranged from 3\% (for all nuclei pooled in the most extensively innervated case) to $14 \%$ (for $\mathrm{Sp} 50$, in the case less densely innervated). The average CEs estimated in varicosity counting ranged from $1.5 \%$ (for all distinct varicosities pooled in each group) to 8\% (for counts in $\mathrm{Sp} 50$ and $\mathrm{Pr} 5$ in groups $\mathrm{C}$ and T). As expected, the CE estimated in TG neuron counting was higher, around $20 \%$

Systematically sampled varicosities from each trigeminal nucleus from three animals of each group were used to estimate varicosity size. Simple inspection of the tissue showed that varicosities that were not spheroidal did not display any preferential orientation. Assuming isotropy, therefore, the isotropic 2D nucleator was used to estimate maximal cross-sectional area. After marking the approximate center in a varicosity, the computer generates four random, right-angled, isotropic directions, and the operator marks the points of intersection of these lines with the periphery of the varicosity. The program computes the length of the intercepts along each test line, and provides an estimate of the area as: $\hat{A}=\pi \cdot \bar{l}^{2}$ (Gundersen 1988; Larsen 1998). Around 200 distinct varicosities were measured per nucleus and case (Table 1).

\section{Additional statistics}

Descriptive statistics for $\mathrm{V}$ and $\mathrm{N}$ per nucleus and group (mean and standard error of the mean) were obtained directly from the Excel spreadsheet (Microsoft Office Professional Plus 2010 for Windows 10, ver. 14.0.7177.5000). For intergroup comparisons the univariate general linear model provided by SPSS (ver. 15.0 for Windows) was used. The homogeneity of variances was assessed with the Levene test. If the global F test showed significant differences, post-hoc corrections were applied using Scheffé or Dunnet's T3 tests. The distributions of distinct CTBlabeled varicosities in four trigeminal nuclei were plotted in Excel, and were compared between group $\mathrm{C}$ and each of the other groups with the two-sample Kolmogorov-Smirnov test (Kirkman 1996). Differences were considered statistically significant when $\mathrm{p}<0.05$.

\section{Results}

\section{Success of tracer delivery}

In order to ascertain to what extent the injected tracers were taken up by axons in the DVN, in several cases we collected and immunostained the TG and a cross section of the C1 DVN $2 \mathrm{~mm}$ proximal to the injection site after 24 hours survival. Axonal labeling in the nerve and neuronal labeling in the ganglion was weak or absent after the customary survival times employed (data 
not shown). We found extensive CTB labeling of myelinated axons in the DVN by immunofluorescence and preembedding immunoelectronmicroscopy. The latter method also showed extensive labeling of unmyelinated axons (Suppl. Fig.1). Neuron bodies in the TG were also labeled from DVN injections, as shown with DAB-based immunocytochemistry and immunofluorescence (Suppl. Fig. 2). The number of axons estimated by stereology in a single naïve nerve was 290, a value close to the mean numbers reported in earlier studies (Crissman et al. 1991). This value matched well with the 318 labeled neurons estimated in the TG in a case with the same $24 \mathrm{~h}$ survival time after DVN injection. It seemed, therefore, that our injection procedure succeeded in delivering the tracers to all axons in the DVN.

Pattern of terminal innervation of trigeminal nuclei in controls

Dense patches of CTB-labeled terminals and varicosities of various sizes, both terminal and en passant, appeared as a rostrocaudally elongated stripe starting at the rostral end of Pr5 and ending in lamina III of Sp5c about $3.5 \mathrm{~mm}$ caudal to the obex (Fig. 2). The stripe was continuous and slender in dorsoventral and mediolateral dimensions in Pr5, Sp5o and Sp5ip. In Sp5c (Fig. 3) the patch was separated from that in Sp5ip by a short unlabeled region, and extended more in both dimensions: rostrally, it was mostly located in lamina IV, with some scattered varicosities in lamina $\mathrm{V}$; caudalwards, it shifted to a more superficial position, mostly occupying the deeper $2 / 3$ of lamina III. In 3 out of 8 control cases a variable amount of small CTB-labeled varicosities could also be found in the inner two-thirds of lamina II. Where the CTB patch reached closer to lamina II, thinner fibers and varicosities immunolabeled with IB4 formed a slender patch, less than $1 \mathrm{~mm}$ long, restricted to lamina I and, mainly, the outer twothirds of lamina II. Both in DAB-revealed and immunofluorescence material, there was a prominent network of CTB-labeled fibers in the outer half of the trigeminal tract (Fig. 3).

Innervation patterns after chronic exposure to enrichment or whisker trimming

No obvious changes with respect to controls were detected at low power in the distribution of CTB- and IB4-labeled profiles in Pr5, Sp5o, Sp5ip or laminae III-IV of Sp5c after unilateral trimming of all vibrissae or exposure to an enriched environment. At higher power, however, CTB-immunoreactive patches appeared denser and more populated with varicosities, particularly in the enriched group. Moreover, the presence of CTB-labeled varicosities in lamina II of Sp5c was manifestly more frequent and abundant when the input was altered, either by sensory deprivation or enrichment (Fig. 4). These varicosities were mostly small, with a minor contribution of medium-sized ones, and were detected in all cases of groups $\mathrm{E}$ and $\mathrm{T}$ ( $n=6$ in each group).

The quantitative analysis (Fig. 5) showed no changes in the volume of the innervated territory in any nucleus in group $\mathrm{T}$, but there was an overall tendency to a greater volume in group $\mathrm{E}$, which reached significance when values for all nuclei were pooled $(\mathrm{p}=0.015)$. The volume of the IB4-immunostained patch remained essentially unchanged in groups $\mathrm{E}$ and $\mathrm{T}$ with respect to controls (Fig. 6). On the other hand, the numbers of distinct varicosities were significantly higher in all nuclei in group $E(\operatorname{Pr} 5: p=0.005$; Sp5o: $p=0.003$; Sp5ip: $p=0.002$; Sp5c: $p=0.003)$, and in Sp5c in group $\mathrm{T}(\mathrm{p}=0.030)$, compared to controls. In the $\mathrm{E}$ group, when all nuclei were pooled together the p-value was less than 0.001 with respect to controls. The mean increases in group E ranged between 64\% in Sp5ip and 120\% in Pr5. In Sp5c, the number of varicosities increased by $88 \%$ and $63 \%$ in groups $\mathrm{E}$ and $\mathrm{T}$, respectively. The pattern of intergroup differences that reached statistical significance did not change when all varicosities (distinct and questionable) were included in the count. No attempt was made to quantify IB4labeled varicosities, because their small size would leave most of them below resolution or 
within a $q$ category, or many could be simply obscured by the diffuse immunostaining of the neuropil.

\section{Colocalization of IB4 and CTB}

Our limited data with confocal microscopy of double immunofluorescent brain stem sections of control cases indicate that none of the CTB-labeled fibers and varicosities in laminae III-IV of Sp5c also contained IB4. Among the limited amount of CTB-labeled profiles that codistributed topographically with IB4-labeled profiles in lamina II, however, in just a few of them an indisputable IB4-CTB colocalization was found, mostly corresponding to fine fibers and small varicosities (Suppl. Fig. 3).

\section{Size of varicosities}

Systematically sampled varicosities were first judged to belong to either the $d$ or the $q$ classes, and then their maximum cross section was measured with the $2 \mathrm{D}$ nucleator. Less than $5 \%$ of all varicosities showed overlapping size estimates between the two classes. A cut-off size of 0.20 $\mu \mathrm{m}^{2}$ was chosen as the minimum cross sectional area for distinct varicosities, since only very rarely one assigned to the $q$ class exceeded that size.

The size distribution in all groups was unimodal and markedly skewed to the right, so that with extreme values differing by two orders of magnitude $\left(0.2\right.$ to $\left.20 \mu \mathrm{m}^{2}\right)$, median values in controls remained at a modest 1.34-1.65 (Table 1). Varicosities in Sp5o displayed similar values irrespective of alterations in the sensory input. On the other hand, the median size of varicosities in other nuclei grew between $18 \%$ and $45 \%$ in groups $\mathrm{E}$ and T over controls. When these distributions were statistically compared with the Kolmogorov-Smirnov test, the results showed a strong and more widespread effect of enrichment, which was followed by significant increases over controls in all nuclei (but Sp5o), while rises in size after whisker trimming only appeared in Sp5ip and laminae III-IV of Sp5c (Fig. 7). Incidentally, the increase in Sp5ip in group $\mathrm{T}$ was significantly smaller $(\mathrm{p}=0.025)$ than in group $\mathrm{E}$ (data not shown).

\section{Discussion}

Using immunocytochemistry and immunofluorescence for simultaneously revealing CTB and IB4, we have been able to successfully trace in the trigeminal nuclei the central terminations of TG neurons whose peripheral axons are contained in a single DVN. Four major outcomes have resulted from this study. First, we have developed a tracer injection technique of the DVN that produced consistent results with a high success rate. Second, we have confirmed earlier descriptions of the regional and laminar pattern of terminal arborizations of physiologically identified vibrissal afferents obtained by intraaxonal HRP or Neurobiotin labeling (Hayashi 1985; Jacquin et al. 1986; Shortland et al. 1995; Shortland et al. 1996). The few comparable tracer studies available for single or multiple DVNs also used HRP, a less sensitive tracer for transganglionic bulk labeling of central afferents. These studies reported a somatotopic distribution of afferents in Pr5, Sp5o, Sp5ip and the deep laminae of Sp5c consistent with our findings, but did not include any quantitative analysis, and failed to notice the afferents in lamina II (Arvidsson 1982; Arvidsson and Johansson 1988; Arvidsson and Rice 1991). Third, we found that the DVN fibers that took up IB4, presumably unmyelinated, distributed exclusively in a restricted sector of laminae I-II in Sp5c. Finally, we have discovered that CTBlabeled varicosities, presumably myelinated, reacted to sustained alterations of sensory input with increases in number and size that were widespread across trigeminal nuclei in the enriched 
condition, while in the whisker trimming condition the effects were restricted to a prominent expansion in both parameters in laminae III-IV of Sp5c. Together with our recent reports on dendritic plasticity in second-order trigeminal neurons (Negredo et al. 2009; Martin et al. 2014), these findings add support to the view that subcortical structures can be modified by experience and may contribute substantially to plasticity at higher levels of the neural system (Kaas et al. 1999).

\section{Methodological limitations}

The simultaneous use of two or more tracers may be detrimental for the successful transganglionic labeling of central projections. For example, Liu et al. (1995) found few DRG ganglion cell bodies labeled, and nearly a total lack of terminal staining in the spinal cord, when combined injections of CTB-HRP and WGA-HRP were made in the sciatic nerve. It seems, however, that no such interference occurs when CTB and other tracers are used in combination. Shehab and Hughes (2011) injected the sciatic nerve with CTB and either IB4 or WGA, and detected in the spinal cord by immunofluorescence a strong labeling of DRG neurons with either tracer, and of myelinated fibers with CTB and unmyelinated fibers with IB4 or WGA.

Although the injection pipette tip is small, even compared with the small caliber of the DVN, some mechanical damage of a number of axons in the nerve cannot be ruled out. It could be argued that the 4-day postinjection survival time we used could be enough to allow for a certain degree of reorganization of the central terminals, so that the "normal" weak innervation of lamina II by CTB-labeled axons, and part of that seen after sensory deprivation of C1, could be attributed to axon damage. This explanation, however, is not supported by available data that showed that a few days after peripheral nerve lesions, the transganglionic transport of different tracers in the spinal cord is essentially unchanged with respect to that seen after similar injections in intact nerves (Woolf et al. 1995; Bao et al. 2002). Therefore, it seems unlikely that the damage inflicted by our injection in the DVN could be responsible for more than a negligible part of the central terminal reorganization.

\section{Effects of haptic deprivation by whisker trimming}

The effects of whisker trimming on higher levels of somatosensory processing, particularly the cortical barrel field, have been extensively investigated not only in postnatal stages, but also in postpuberal and fully mature subjects (Fuchs and Salazar 1998; Maier et al. 2003; Machin et al. 2006; Dolan and Cahusac 2007; Katzel and Miesenbock 2014). On the other hand, with few exceptions, the effects of sensory deprivation on the lower levels of sensory processing have not been examined in mature mammals. Second order neurons in the trigeminal pathways display dendritic morphometric changes following chronic manipulations of their sensory inputs (Negredo et al. 2009; Martin et al. 2014), and more recently, we found that a number of genes and proteins related to glutamatergic transmission change their expression in the trigeminal ganglion of adult rats after chronic deprivation by unilateral whisker trimming (Fernandez-Montoya et al. 2016). The effects in this study were bilateral, with substantial increases in the expression of genes coding for several AMPA, NMDA and metabotropic receptor subunits in the deprived ganglion, and decreases in the contralateral TG. In the auditory system, a recent study in young adult rats (Clarkson et al. 2016) showed that ten days of unilateral auditory deprivation by ear plugging brought about structural and molecular changes in the synaptic terminals of the auditory nerve on the cochlear nucleus. These changes consisted of a decrease in the number, density and size of synaptic vesicles, and in the expression of a vesicular transporter of glutamate, and were associated to a longer-lasting 
thickening of the postsynaptic density and a changed subunit composition of AMPA receptors. The authors suggested that these effects may reflect a compensatory potentiation of synaptic responses associated to a reduction of sensory input in the primary fiber.

These findings may be in consonance with our present results. Using the same whisker trimming protocol, we reported recently that deprived intersubnuclear neurons in Sp5c displayed a significant reduction in the number of spines and complex dendritic arbors, and a moderate lengthening of some dendritic segments (Martin et al. 2014). The disappearance of a normally patterned sensory input may have induced an expansion, in terms of number and size, of putative presynaptic structures, in turn resulting in morphometric changes in postsynaptic dendrites in Sp5c. It is intriguing, however, that a similar phenomenon is not found in other nuclei, such as Pr5 and Sp5o, while in Sp5ip varicosities appeared to be larger, but not more numerous. It is unknown whether specific afferents concerning fiber type and receptor origin in the follicle, coursing within the DVN have preferential or even separate targets within the trigeminal sensory complex. Should such segregation exist, one could wonder whether deprivation of haptic touch would differentially affect DVN fibers, and hence elicit a heterogeneous pattern of structural changes in the complex. While still speculative, this possibility could find support in previous reports that describe distinctive morphological features of low- and high-threshold primary afferent endings in laminae III-V of Sp5c (Jacquin et al. 1986; Miyoshi et al. 1994), although the differences found by others were considered unremarkable (Jacquin et al. 1984; Hayashi 1985).

\section{Effects of exposure to sensory enrichment}

Since Hebb proposed that rats living in naturalistic, rich sensorimotor environments learned to run mazes better than rats kept in standard laboratory cages (Hebb 1949), many studies have shown that adult rats exposed to enriched environments exhibit structural and functional changes in a number of brain regions and systems. Because of its accessibility and behavioral relevance, the trigeminal system in rodents has been a frequent target for these studies. In rodents exposed to this type of environment, the somatosensory cortex exhibits increases in cortical thickness and volume, dendrite number and length, and number and/or size of spines, synapses, glial cells and capillaries (Diamond 1988; Machín et al. 2004; Nithianantharajah et al. 2004; Rema et al. 2006; Landers et al. 2011), as well as refinements of topographic maps and potentiation of sensory responses (Frostig 2006; Devonshire et al. 2010).

The induction of synaptic strengthening by experience has been extensively documented in the cortex, and has been considered a critical neurobiological substrate for experiencedependent plasticity, learning and memory (Fox and Wong 2005; Holtmaat and Svoboda 2009; Cheetham et al. 2012; Froemke 2015). Changes in synaptic strength have also been linked to modifications in the structure of synapses (Federmeier et al. 2002; Cheetham et al. 2012), and synaptogenesis and increase in the number of synaptic contacts have been described as a neural substrate of motor learning (Black et al. 1990; Anderson et al. 1996; Kleim et al. 2004) and a general feature of circuit remodeling in response to experience (Gogolla et al. 2007).

The effects of environmental enrichment on primary somatosensory neurons have not been examined heretofore, but for a recent study (Fernandez-Montoya et al. 2016) that reported substantial changes in the expression in TG of a number of genes and proteins related to glutamatergic transmission. In the present study, we found an extensive increase in number and size of varicosities after enrichment, but we cannot elucidate whether the numerical increase in all trigeminal nuclei is due to an actual appearance of new varicosities, to the enlargement of small, previously existing varicosities that would bring them above the detection threshold, or to both processes. It seems clear, nevertheless, that the enlargement of presumed presynaptic boutons should bear functional repercussions, since bouton volume correlates with the area and 
even the number of active zones in it, and with the probability of neurotransmitter release (Nakagawa et al. 1997; Murthy et al. 2001; Federmeier et al. 2002; Cheetham et al. 2012; Kitahara et al. 2016).

It is likely that the effects of enrichment on the morphometry of varicosities are not only presynaptic, but result from an interaction between pre- and postsynaptic elements. In first place, because the enlargement of varicosities did not occur in all nuclei despite the similarity of afferents: varicosities increased in size in Pr5 and failed to do so in Sp5o, when the morphologies of terminal arbors in these nuclei were indistinguishable (Jacquin et al. 1993). As previously discussed for the deprivation condition, it may be suggested that the various primary afferents that synapse on different neurons in each nucleus may have been affected differently by exposure to enrichment. Afferents to the vibrissa-representing barrelette sector in Pr5 receive most of their peripheral input from whisker follicles, while neurons in Sp5o display a much greater morphological diversity, notable convergence of input from a variety of receptor types and orofacial regions, and characteristic response properties (Jacquin and Rhoades 1990; Huopaniemi et al. 1992). It could then be predicted that neurons more heavily impacted by vibrissa afferents will be more affected by changes in input that particularly involve vibrissae. Through retrograde signaling, the postsynaptic neuron could then influence a specific set of its presynaptic boutons towards generating more or more efficient synaptic contacts (Gogolla et al. 2007).

Effects of input alteration on the dual innervation of Sp5c

It is common for any single vibrissal or non-vibrissal skin afferent to issue collaterals along their course that leave a number of terminal patches in lamina $\mathrm{V}$ more rostrally, and in laminae III and IV in more caudal parts of Sp5c (Hayashi 1980; Hayashi 1982; Hayashi 1985; Jacquin et al. 1986; Shortland et al. 1995). In contrast, unmyelinated C-fibers from the facial skin distribute exclusively in laminae I-II of Sp5c (Kobayashi and Matsumura 1996).

In the spinal dorsal horn, the laminar pattern of primary afferent terminals is known to be altered by different injuries to the peripheral nerves. Following nerve damage, myelinated primary afferents normally innervating only laminae I and III-V but sparing lamina II, were found in large numbers in the latter. Since CTB is a well-established marker of large primary sensory neurons, this observation was initially interpreted as a sprouting response of $A \beta$-fibers into lamina II (Coggeshall et al. 1997; Koerber et al. 1994; Shortland et al. 1997; Woolf et al. 1992; Woolf et al. 1995). Nevertheless, some studies reported a few small-sized cell bodies in the DRG labeled by CTB, as well as a small amount of CTB labeling as well in lamina II in controls (Shehab and Hughes 2011; Tong et al. 1999; Woolf et al. 1995). Other laboratories, however, offered evidence against this mechanism of translaminar central sprouting of A-fibers after nerve lesions, interpreting these findings as the result of a phenotypic switch in $\mathrm{C}$ afferents, which after injury would express in their membrane the GM1 monoganglioside, and hence the ability to take up and transport CTB (Tong et al. (1999). A variety of more recent tracing studies supported this contention (Santha et al. 2003; Shehab et al. 2003; Woodbury et al. 2008; Oszlacs et al. 2015). The only comparable study in the IoN territory, which studied the terminals from genetically labeled, parvalbumin-expressing $\mathrm{A} \beta$-neurons of the trigeminal ganglion, also failed to support the thesis of reactive sprouting in lamina II of Sp5c following inflammation or transection of the nerve (Zhang et al. 2015).

Although unable to exclude the existence of some degree of sprouting of CTB-labeled A $\beta$ fibers, at least in the inner half of lamina II, our findings favor the option of IB4-labeled Cfibers acquiring the capacity to take up CTB, since most of the labeled profiles in lamina II correspond to small varicosities and fine axonal profiles. Our limited data on IB4-CTB colabeling also support this view. But our findings add to this matter the unexpected observation 
that such phenotypic switch may take place in absence of any nerve injury, suggesting that this phenomenon may play a more general role in experience-dependent plasticity.

\section{Acknowledgements}

The authors gratefully acknowledge Ms. Begoña Rodriguez for her skilled technical help in the preparation of the histological material, and the Servicios de Microscopía Confocal and Microscopía Electrónica de Transmisión of the SIDI-UAM for their help in the confocal microscopy analyses. We also thank Dr. A. Krzyzanowska for reading a final draft of the manuscript and making useful style suggestions. This study was supported by Grants BFU2012-39960 and BFU2015-66941R from Spain's Ministerio de Economía Industria y competitividad / Fondo Europeo de Desarrollo Regional (MINECO/FEDER). The authors have no conflicts of interest. 


\section{References}

Aldskogius H, Arvidsson J, Grant G (1985) The reaction of primary sensory neurons to peripheral nerve injury with particular emphasis on transganglionic changes. Brain Res Rev 10:27-46

Ambalavanar R, Morris R (1993) An ultrastructural study of the binding of an alpha-Dgalactose specific lectin from Griffonia simplicifolia to trigeminal ganglion neurons and the trigeminal nucleus caudalis in the rat. Neuroscience 52:699-709

Anderson RL, Gibbins IL, Morris JL (1996) Non-noradrenergic sympathetic neurons project to extramuscular feed arteries and proximal intramuscular arteries of skeletal muscles in guinea-pig hindlimbs. J Auton Nerv Syst 61:51-60

Arvidsson J (1982) Somatotopic organization of vibrissae afferents in the trigeminal sensory nuclei of the rat studied by transganglionic transport of HRP. J Comp Neurol 211:84-92

Arvidsson J, Johansson K (1988) Changes in the central projection pattern of vibrissae innervating primary sensory neurons after peripheral nerve injury in the rat. Neurosci Lett 84:120-124

Arvidsson J, Rice FL (1991) Central projections of primary sensory neurons innervating different parts of the vibrissae follicles and intervibrissal skin on the mystacial pad of the rat. J Comp Neurol 309:1-16

Avendaño C (2006) Stereology of neural connections. An overview. In: Záborszky L et al. (eds) Neuroanatomical Tract Tracing: Molecules, Neurons, \& Systems, 3 edn. Springer Science \& Business Media, pp 477-529

Bao L, Wang HF, Cai HJ, Tong YG, Jin SX, Lu YJ, Grant G, Hokfelt T, Zhang X (2002) Peripheral axotomy induces only very limited sprouting of coarse myelinated afferents into inner lamina II of rat spinal cord. Eur J Neurosci 16:175-185

Bjelke K, Aldskogius H, Arvidsson J (1996) Short- and long-term transganglionic changes in the central terminations of transected vibrissal afferents in the rat. Exp Brain Res 112:268276

Black JE, Isaacs KR, Anderson BJ, Alcantara AA, Greenough WT (1990) Learning causes synaptogenesis, whereas motor activity causes angiogenesis, in cerebellar cortex of adult rats. Proc Nat Acad Sci USA 87:5568-5572

Brown AG (1981) Organization in the Spinal Cord. The Anatomy and Physiology of Identified Neurones. Springer-Verlag, Berlin

Bush NE, Solla SA, Hartmann MJ (2016) Whisking mechanics and active sensing. Curr Opin Neurobiol 40:178-188

Cheetham CE, Barnes SJ, Albieri G, Knott GW, Finnerty GT (2012) Pansynaptic Enlargement at Adult Cortical Connections Strengthened by Experience. Cereb Cortex 24:521-531

Clarkson C, Antunes FM, Rubio ME (2016) Conductive Hearing Loss Has Long-Lasting Structural and Molecular Effects on Presynaptic and Postsynaptic Structures of Auditory Nerve Synapses in the Cochlear Nucleus. J Neurosci 36:10214-10227

Coggeshall RE, Lekan HA, Doubell TP, Allchorne A, Woolf CJ (1997) Central changes in primary afferent fibers following peripheral nerve lesions. Neuroscience 77:1115-1122 
Crissman RS, Warden RJ, Siciliano DA, Klein BG, Renehan WE, Jacquin MF, Rhoades RW (1991) Numbers of axons innervating mystacial vibrissa follicles in newborn and adult rats. Somatosens Motor Res 8:103-109

Cruz-Orive LM (1999) Precision of Cavalieri sections and slices with local errors. J Microsc 193:182-198

Devonshire IM, Dommett EJ, Grandy TH, Halliday AC, Greenfield SA (2010) Environmental enrichment differentially modifies specific components of sensory-evoked activity in rat barrel cortex as revealed by simultaneous electrophysiological recordings and optical imaging in vivo. Neuroscience 170:662-669

Diamond MC (1988) Enriching Heredity. The impact of the environment on the anatomy of the brain. The Free Press, London

Dolan S, Cahusac PM (2007) Enhanced short-latency responses in the ventral posterior medial (VPM) thalamic nucleus following whisker trimming in the adult rat. Physiol Behav 92:500-506

Ebner FF (2005) Neural Plasticity in Adult Somatic Sensory-Motor Systems. Taylor \& Francis/CRC Press, Boca Raton, FL

Federmeier KD, Kleim JA, Greenough WT (2002) Learning-induced multiple synapse formation in rat cerebellar cortex. Neurosci Lett 332:180-184

Fernandez-Montoya J, Buendia I, Martin YB, Egea J, Negredo P, Avendano C (2016) Sensory Input-Dependent Changes in Glutamatergic Neurotransmission- Related Genes and Proteins in the Adult Rat Trigeminal Ganglion. Front Mol Neurosci 9:132. eCollection@2016:132

Florence SL, Kaas JH (1995) Large-scale reorganization at multiple levels of the somatosensory pathway follows therapeutic amputation of the hand in monkeys. $\mathbf{J}$ Neurosci 15:8083-8095

Fox K, Wong RO (2005) A comparison of experience-dependent plasticity in the visual and somatosensory systems. Neuron 48:465-477

Froemke RC (2015) Plasticity of cortical excitatory-inhibitory balance. Annu Rev Neurosci 38:195-219

Frostig RD (2006) Functional organization and plasticity in the adult rat barrel cortex: moving out-of-the-box. Curr Opin Neurobiol 16:445-450

Fuchs JL, Salazar E (1998) Effects of whisker trimming on GABAA receptor binding in the barrel cortex of developing and adult rats. J Comp Neurol 395:209-216

Gogolla N, Galimberti I, Caroni P (2007) Structural plasticity of axon terminals in the adult. Curr Opin Neurobiol 17:516-524

Gundersen HJG (1988) The nucleator. J Microsc 151:3-21

Gundersen HJG, Bendtsen TF, Korbo L, Marcussen N, Møller A, Nielsen K, Nyengaard JR, Pakkenberg B, Sørensen FB, Vesterby A, West MJ (1988) Some new, simple and efficient stereological methods and their use in pathological research and diagnosis. APMIS 96:379394

Hayashi H (1980) Distributions of vibrissae afferent fiber collaterals in the trigeminal nuclei as revealed by intra-axonal injection of horseradish peroxidase. Brain Res 183:442-446

Hayashi H (1982) Differential terminal distribution of single large cutaneous afferent fibers in the spinal trigeminal nucleus and in the cervical spinal dorsal horn. Brain Res 244:173-177 
Hayashi H (1985) Morphology of central terminations of intra-axonally stained, large, myelinated primary afferent fibers from facial skin in the rat. J Comp Neurol 237:195-215

Hebb DO (1949) The organization of behavior. A neuropsychological theory. Wiley, New York

Holtmaat A, Svoboda K (2009) Experience-dependent structural synaptic plasticity in the mammalian brain. Nat Rev Neurosci 10:647-658

Huopaniemi T, Jyvasjarvi E, Carlson S, Lindroos F, Pertovaara A (1992) Response characteristics of tooth pulp-driven postsynaptic neurons in the spinal trigeminal subnucleus oralis of the cat. Acta Physiol Scand 144:177-183

Jacquin MF, Mooney RD, Rhoades RW (1984) Axon arbors of functionally distinct whisker afferents are similar in medullary dorsal horn. Brain Res 298:175-180

Jacquin MF, Renehan WE, Mooney RD, Rhoades RW (1986) Structure-function relationships in rat medullary and cervical dorsal horns. I. Trigeminal primary afferents. J Neurophysiol 55:1153-1186

Jacquin MF, Renehan WE, Rhoades RW, Panneton WM (1993) Morphology and topography of identified primary afferents in trigeminal subnuclei principalis and oralis. $\mathrm{J}$ Neurophysiol 70:1911-1936

Jacquin MF, Rhoades RW (1990) Cell structure and response properties in the trigeminal subnucleus oralis. Somatosens Motor Res 7:265-288

Kaas JH, Florence SL, Jain N (1999) Subcortical contributions to massive cortical reorganizations. Neuron 22:657-660

Katzel D, Miesenbock G (2014) Experience-dependent rewiring of specific inhibitory connections in adult neocortex. PLoS Biol 12:e1001798

Kirkman TW (1996) Statistics to Use. p 1-9.

Kitahara Y, Ohta K, Hasuo H, Shuto T, Kuroiwa M, Sotogaku N, Togo A, Nakamura K, Nishi A (2016) Chronic Fluoxetine Induces the Enlargement of Perforant Path-Granule Cell Synapses in the Mouse Dentate Gyrus. PLoS ONE 11:e0147307

Kleim JA, Hogg TM, VandenBerg PM, Cooper NR, Bruneau R, Remple M (2004) Cortical synaptogenesis and motor map reorganization occur during late, but not early, phase of motor skill learning. J Neurosci 24:628-633

Kleinfeld D (2009) Vibrissa Movement, Sensation and Sensorimotor Control. Academic Press; p 155-77.

Kobayashi Y, Matsumura G (1996) Central projections of primary afferent fibers from the rat trigeminal nerve labeled with isolectin B4-HRP. Neurosci Lett 217:89-92

Koerber HR, Mirnics K, Brown PB, Mendell LM (1994) Central sprouting and functional plasticity of regenerated primary afferents. J Neurosci 14:3655-3671

LaMotte CC, Kapadia SE, Shapiro CM (1991) Central projections of the sciatic, saphenous, median, and ulnar nerves of the rat demonstrated by transganglionic transport of choleragenoid-HRP (B-HRP) and wheat germ agglutinin- HRP (WGA-HRP). J Comp Neurol 311:546-562

Landers MS, Knott GW, Lipp HP, Poletaeva I, Welker E (2011) Synapse formation in adult barrel cortex following naturalistic environmental enrichment. Neuroscience 199:143-152 Larsen JO (1998) Stereology of nerve cross sections. J Neurosci Methods 85:107-118 
Liu H, Llewellyn-Smith IJ, Basbaum AI (1995) Co-injection of wheat germ agglutinin-HRP and choleragenoid-HRP into the sciatic nerve of the rat blocks transganglionic transport. J Histochem Cytochem 43:489-495

Machín R, Blasco B, Bjugn R, Avendaño C (2004) The size of the whisker barrel field in adult rats: minimal nondirectional asymmetry and limited modifiability by chronic changes of the sensory input. Brain Res 1025:130-138

Machin R, Perez-Cejuela CG, Bjugn R, Avendano C (2006) Effects of long-term sensory deprivation on asymmetric synapses in the whisker barrel field of the adult rat. Brain Res 1107:104-110

Maier DL, Grieb GM, Stelzner DJ, McCasland JS (2003) Large-scale plasticity in barrel cortex following repeated whisker trimming in young adult hamsters. Exp Neurol 184:737-745

Martin YB, Negredo P, Villacorta-Atienza JA, Avendano C (2014) Trigeminal intersubnuclear neurons: morphometry and input-dependent structural plasticity in adult rats. J Comp Neurol 522:1597-1617

Miyoshi Y, Suemune S, Yoshida A, Takemura M, Nagase Y, Shigenaga Y (1994) Central terminations of low-threshold mechanoreceptive afferents in the trigeminal nuclei interpolaris and caudalis of the cat. J Comp Neurol 340:207-232

Murthy VN, Schikorski T, Stevens CF, Zhu Y (2001) Inactivity produces increases in neurotransmitter release and synapse size. Neuron 32:673-682

Nakagawa S, Kurata S, Yoshida A, Nagase Y, Moritani M, Takemura M, Bae YC, Shigenaga Y (1997) Ultrastructural observations of synaptic connections of vibrissa afferent terminals in cat principal sensory nucleus and morphometry of related synaptic elements. J Comp Neurol 389:12-33

Negredo P, Martin YB, Lagares A, Castro J, Villacorta JA, Avendano C (2009) Trigeminothalamic barrelette neurons: natural structural side asymmetries and sensory input-dependent plasticity in adult rats. Neuroscience 163:1242-1254

Nithianantharajah J, Levis H, Murphy M (2004) Environmental enrichment results in cortical and subcortical changes in levels of synaptophysin and PSD-95 proteins. Neurobiol Learn Mem 81:200-210

Oszlacs O, Jancso G, Kis G, Dux M, Santha P (2015) Perineural capsaicin induces the uptake and transganglionic transport of choleratoxin B subunit by nociceptive C-fiber primary afferent neurons. Neuroscience 311:243-252

Paxinos G, Watson C (1998) The Rat Brain in Stereotaxic Coordinates. Academic Press, New York

Rema V, Armstrong-James M, Jenkinson N, Ebner FF (2006) Short exposure to an enriched environment accelerates plasticity in the barrel cortex of adult rats. Neuroscience 140:659672

Rice FL, Mance A, Munger BL (1986) A comparative light microscopic analysis of the sensory innervation of the mystacial pad. I. Innervation of vibrissal follicle-sinus complexes. $\mathrm{J}$ Comp Neurol 252:154-174

Robertson B, Perry MJ, Lawson SN (1991) Populations of rat spinal primary afferent neurons with choleragenoid binding compared with those labelled by markers for neurofilament and carbohydrate groups: a quantitative immunocytochemical study. J Neurocytol 20:387395 
Santha P, Jancso G (2003) Transganglionic transport of choleragenoid by capsaicin-sensitive C-fibre afferents to the substantia gelatinosa of the spinal dorsal horn after peripheral nerve section. Neuroscience 116:621-627

Shehab SAS, Hughes DI (2011) Simultaneous identification of unmyelinated and myelinated primary somatic afferents by co-injection of isolectin B4 and Cholera toxin subunit B into the sciatic nerve of the rat. J Neurosci Methods 198:213-221

Shehab SAS, Spike RC, Todd AJ (2003) Evidence against cholera toxin B subunit as a reliable tracer for sprouting of primary afferents following peripheral nerve injury. Brain Res 964:218-227

Shortland P, Kinman E, Molander C (1997) Sprouting of A-fibre primary afferents into lamina II in two rat models of neuropathic pain. Eur J Pain 1:215-227

Shortland PJ, Demaro JA, Jacquin MF (1995) Trigeminal structure-function relationships: a reevaluation based on long-range staining of a large sample of brainstem A beta fibers. Somatosens Motor Res 12:249-275

Shortland PJ, Demaro JA, Shang F, Waite PME, Jacquin MF (1996) Peripheral and central predictors of whisker afferent morphology in the rat brainstem. J Comp Neurol 375:481501

Silverman JD, Kruger L (1990) Selective neuronal glycoconjugate expression in sensory and autonomic ganglia: relation of lectin reactivity to peptide and enzyme markers. $\mathrm{J}$ Neurocytol 19:789-801

Tong YG, Wang HF, Ju G, Grant G, Hökfelt T, Zhang X (1999) Increased uptake and transport of cholera toxin B-subunit in dorsal root ganglion neurons after peripheral axotomy:

Possible implications for sensory sprouting. J Comp Neurol 404:143-158

West MJ, Slomianka L, Gundersen HJG (1991) Unbiased stereological estimation of the total number of neurons in the subdivisions of the rat hippocampus using the optical fractionator. Anat Rec 231:482-497

Willis WDJ, Coggeshall RE (2004) Sensory Mechanisms of the Spinal Cord. Vol. 1. Primary Afferent Neurons and the Spinal Dorsal Horn. Kluwer/Plenum, New York

Woodbury CJ, Kullmann FA, McIlwrath SL, Koerber HR (2008) Identity of myelinated cutaneous sensory neurons projecting to nocireceptive laminae following nerve injury in adult mice. J Comp Neurol 508:500-509

Woolf CJ, Shortland P, Coggeshall RE (1992) Peripheral nerve injury triggers central sprouting of myelinated afferents. Nature 355:75-78

Woolf CJ, Shortland P, Reynolds M, Ridings J, Doubell T, Coggeshall RE (1995)

Reorganization of central terminals of myelinated primary afferents in the rat dorsal horn following peripheral axotomy. J Comp Neurol 360:121-134

Zhang Y, Chen Y, Liedtke W, Wang F (2015) Lack of evidence for ectopic sprouting of genetically labeled Abeta touch afferents in inflammatory and neuropathic trigeminal pain. Mol Pain 11:18-0017 


\section{Figure Legends}

Fig. 1 Examples of CTB-labeled varicosities in Sp5c. Medium-sized and large varicosities are easily recognized and made the largest contribution to the population of varicosities identified as distinct. Among the small-sized labeled profiles were some that corresponded to obvious swellings along thin axons (some examples are pointed by filled arrows). Isolated profiles of similar sizes were separately identified as questionable, as were very small profiles apparently associated to very thin axons (empty arrows). Other very small dot-like profiles scattered across the neuropil were not included in the count (some examples indicated by arrowheads). Scale bar $10 \mu \mathrm{m}$

Fig. 2 Distribution of projection fields in the brain stem trigeminal nuclei of CTBimmunolabeled axon terminals from the $\mathrm{C} 1 \mathrm{DVN}$ in a control case. Labeling is shown on every second horizontal section, and contrast and brightness were adjusted to enhance visualization. The approximate dorsoventral location of each section is indicated in the inset (lower left corner), and its stereotaxic horizontal position is given by Paxinos and Watson's (1998) coordinates above the sections. On the right, a horizontal section stained for cytochrome oxidase $(\mathrm{CyO})$ from a separate study is shown. This section approximately matches the orientation of the sections with CTB labeling. Arrowheads mark approximate borders between nuclei.

Fig. 3 Patterns of terminal innervation in Sp5c of CTB- and IB4-labeled afferents from C1 DVN in a control case (a-c), in a case with whisker trimming (d-f), and in a case subjected to enrichment (g-h). In horizontal sections of the medulla a laminar pattern is found with double immunofluorescence for CTB (a, red) and IB4 (b, green; c, merged image). Dense CTBlabeling is easily identifiable in the trigeminal tract and in laminae III-IV, while IB4-labeled terminals are restricted to lamina II. Sparse CTB-labeling is also seen in laminae I-II, occasionally marking a continuation from tract fibers into terminal fields in lamina III. The same global pattern is found in other groups, and when the immunostaining is revealed with DAB. CTB-labeled terminals form a dense patch in laminae IV (more rostrally, as in $\mathbf{d}$ and left part of $\mathbf{g}$ ) and III (more caudally, e and right part of $\mathbf{g}$ ). IB4-labeled fibers concentrate in lamina II, more intensely in its outer two-thirds $(\mathbf{f}, \mathbf{h})$. Arrowheads mark laminar boundaries. Scale bars $100 \mu \mathrm{m}$.

Fig. 4 Samples of CTB-labeled terminal axons and varicosities in laminae I and II of Sp5c in controls (C) and experimental (E and T) groups. Details at higher power (areas marked by rectangles on top panels) are shown below. The control case corresponds to one with the densest innervation of lamina II in this group. Examples for $\mathrm{E}$ and $\mathrm{T}$ are taken from representative cases in each group. Dashed lines roughly outline lamina I; the trigeminal tract appears on top, and lamina II is below. Scale bars $20 \mu \mathrm{m}$ (top) and $10 \mu \mathrm{m}$ (bottom).

Fig. 5 Volume of neuropil innervated by primary afferent terminals from C1 DVN in millions of cubic microns (top), and total number of distinct varicosities in thousands (bottom). Values correspond to means+sem and are color-coded for groups control $(C$, blue; $n=8)$, enriched (E, magenta; $n=6)$ and Trimming $(T$, green; $n=6)$. Statistically significant differences represent $0.01<\mathrm{p}<0.05(*)$ and $\mathrm{p}<0.005(* *) . \mathrm{C}_{2}$, lamina II of the caudal division of the spinal nucleus; $\mathrm{C}_{3}$, laminae III and IV of the caudal division of the spinal nucleus; Ip, interpolar nucleus; Or, oral nucleus; Pr, principal nucleus. 
Fig. 6 Volume of neuropil innervated by IB4-immunostained terminals from C1 DVN in millions of cubic microns (left). The patch of labeling was restricted to lamina II in Sp5c, as shown at its maximum spread in a case from group $\mathrm{T}$ (right). Values correspond to means+sem and are color-coded for groups as in Fig. 5. No significant differences were found between groups. Scale bar $500 \mu \mathrm{m}$.

Fig. 7 Distribution of distinct CTB-labeled varicosities in four trigeminal nuclei, grouped into sixteen classes according to their maximal cross-sectional area. Classes 2-15 represent increasing sizes between 1.1 and 15 square microns, at one square micron steps increments. Class 1 collects varicosities measuring 0.2-1.0 square microns. Curves represent mean \pm sem values of three representative cases of each group, which is color-coded as in Fig. 5 (C, blue; E, magenta; $\mathrm{T}$, green). Distributions were compared between group $\mathrm{C}$ and each of the other groups with the Kolmogorov-Smirnov test. When differences were significant $(\mathrm{p}<0.05)$, the cumulative frequency plot is shown as an inset.

\section{Table 1}

Descriptive statistics of the cross-sectional size of $d$ istinct CTB-labeled varicosities in the trigeminal nuclei.

\begin{tabular}{|c|c|c|c|c|c|c|c|c|c|}
\hline & \multicolumn{3}{|c|}{$\mathrm{N}$ varicosities examined ${ }^{\mathrm{a}}$} & \multicolumn{3}{|c|}{ Mean $\left(\mu \mathrm{m}^{2}\right)$} & \multicolumn{3}{|c|}{$\operatorname{Median}\left(\mu \mathrm{m}^{2}\right)$} \\
\hline & $\mathrm{C}$ & $\mathrm{E}$ & $\mathrm{T}$ & $\mathrm{C}$ & $\mathrm{E}$ & $\mathrm{T}$ & $\mathrm{C}$ & $\mathrm{E}$ & $\mathrm{T}$ \\
\hline $\operatorname{Pr} 5$ & 619 & 593 & 642 & 2.22 & 2.80 & 2.48 & 1.64 & 1.93 & 1.93 \\
\hline Sp5o & 627 & 422 & 689 & 2.14 & 2.15 & 2.12 & 1.62 & 1.61 & 1.62 \\
\hline Sp5ip & 438 & 678 & 723 & 1.82 & 2.50 & 2.51 & 1.34 & 1.94 & 1.87 \\
\hline $\mathrm{Sp} 5 \mathrm{c}$ & 578 & 759 & 717 & 2.06 & 2.44 & 2.63 & 1.65 & 1.95 & 2.12 \\
\hline
\end{tabular}

a Pooled from three animals per group

\section{Supplementary material}

Figure 1:Injection of CTB and IB4 in a single deep vibrissal nerve (DVN). (a) Exposure of the deep nerve from vibrissal follicle $\mathrm{C} 1$. Star, follicle; arrows, stretch of the nerve attaching to the follicle wall. (b) Intraneural deposit of a mixture of tracers with Light Green to help visualization of the same nerve; the picture was taken immediately after finishing the injection. (c) Confocal immunofluorescence image of a cross section of C1 DVN, $2 \mathrm{~mm}$ proximal to the injection. Most, if not all, medium-sized and large axons are labeled by CTB (red). Asterisk marks a blood vessel artifact. (d) Semithin section of C1 DVN injected with both tracers, subjected to a preembedding immunohistochemistry and counterstained with toluidine blue. The axoplasm of most myelinated axons, normally unstained by toluidine blue, exhibit now a dark color due to the immunoreaction. (e) Low power electron micrograph of a cross section of C1 DVN in a naïve case without injection of tracers. (f, $\mathbf{g})$ High power electron micrographs of the same C1 DVN as in (d), showing details of several labeled myelinated and unmyelinated axons. The axoplasm of myelinated axons seems uniformly filled with a diffuse precipitate 
which masks the axonal cytoskeleton. Unmyelinated axons are irregularly filled with dark deposits (arrows). Scale bars $200 \mu \mathrm{m}$ (a,b), $20 \mu \mathrm{m}$ (c), $50 \mu \mathrm{m}$ (d,e), $2 \mu \mathrm{m}$ (f,g).

Figure 2: Panels showing examples of neuron somata in the TG of a control case that are single-labeled by either CTB (a, b, c: red, left boxes of each panel) or IB4 (a, b, c: green, middle boxes). Merged images in the boxes on the right show no colocalization of the tracers. In (c) however, a few small neurons in the same case show colocalization (arrowheads). Scale bars: $40 \mu \mathrm{m}$.

Figure 3: Confocal merged images with orthogonal projections showing three examples of varicosities double-labeled for CTB (red) and IB4 (green) in lamina II of Sp5c in a control case. (a) Example of a fairly large double-labeled varicosity (arrow) located very close to another varicosity that is only labeled for CTB. (b,c) Crosshairs marking two small doublelabeled profiles, probably corresponding to small varicosities. Scale bars $50 \mu \mathrm{m}(\mathbf{a}), 10 \mu \mathrm{m}(\mathbf{b}$, c). 